\title{
Research and application of multi hydracid multilevel hierarchical acidification
}

\author{
Zhixiang Zhu ${ }^{1, a}$, Wenying Zhang ${ }^{2, b}$ \\ ${ }^{1}$ Production Engineering Research Institute, Huabei Oilfield Branch(CNPC), Cangzhou 062552, \\ China \\ ${ }^{2}$ Production Engineering Research Institute, Huabei Oilfield Branch(CNPC), Cangzhou 062552, \\ China \\ aemail: cyy_zzx@petrochina.com.cn, bemail: cyy_zwy@petrochina.com.cn
}

Keywords: Multi-hydrogen acid; Layered acidification; Low permeability reservoir; decompression and stimulate

\begin{abstract}
M31 block of sandstone reservoirs, with strong heterogeneity and poor physical property, belongs to ultra-low permeability reservoirs, and the reservoir permeability is around $9 \mathrm{mD}$. The clay mineral content exceeds $5 \%$, and the daily water capacity can't meet the requirements of injection allocation. Reservoir near Wells is contaminated, and the oil and liquids production of corresponding wells is very low. For ultra-low permeability sandstone reservoir characteristics, a new type of acidification process called Multi hydracid multilevel hierarchical Acidification is obtained through laboratory study. This process can overcome limitations of the conventional acidification such as small effective role radius and acidified precipitate secondary pollution, and can avoid the effect of different reservoirs between acidification, to effectively improve the water injection profile. The application of the new process in Well M31-6 field shows that hydrogen-acid multilevel hierarchical acidification process is simple and practical, and can reduce water injection pressure and increase water capacity significantly, which has an important reference for the future development of ultra-low permeability sandstone reservoirs and enhancing the final oil recovery.
\end{abstract}

\section{Introduction}

M31 block has characteristics of strong heterogeneity, poor physical property, low porosity and permeability; the daily water capacity can not meet the requirements of injection allocation , every day is difficult to achieve with injection water injection requirements, and reservoir near Wells is contaminated. If using conventional acidification to sour reservoir, repeated construction is needed, with long cycle and high cost; and repeatedly killing also cause secondary pollution ${ }^{[1]}$. So the multi hydracid multilevel hierarchical Acidification process was researched using respective design and unified solution method.

Multi hydracid multilevel hierarchical acidification is a special acidification process developed on the basis of conventional acidification process ${ }^{[2]}$, with advantage of slow response, strong solvency, good antifouling properties and dispersion properties. It can inhibit the formation damage near the wellbore, and effectively control the secondary sedimentation, etc. The multilevel hierarchical acidification string is simply operated, convenient and practical. Through the multistage packer and sleeve switch, the string is able to separate a plurality of layers, and respectively squeeze targeted acid system and the amount of pollution in accordance with the causes and extent of oil pollution in different strata, thus effectively improving the absorption profile, increase the effective water and so on.

Field application results show that the process of acidification effect is obvious, profile improved significantly, low permeability, multi-department, cross-sectional view of the improved heterogeneous reservoirs provides an effective means of technology, it is recommended to increase efforts to promote the application. 


\section{M31-6 survey}

M31 belt structure located in the ancient hill drape Tertiary-relief anticline belt, buried bedrock Wumishan formation and development over the erosion surface Kongdian, Shahejie Formation, Dongying groups, etc. Drape structural layer, reservoir layer mainly lacustrine deposits.

Light gray sandstone reservoir, with gray-brown sandy mudstone, mudstone quality pure, brittle and hard, calcium weak; sandstone composition dominated by quartz, feldspar followed, containing a small amount of dark minerals. Reservoir porosity of $12.2 \% \sim 15.4 \%$, the average air permeability values $(0.1 \sim 2.7) \times 10^{-3} \mu \mathrm{m}^{2}$, oil saturation of $35 \% \sim 67.2 \%$, it has a sandstone moderate acid-sensitive, strong side water sensitivity, strong middle salt-sensitive characteristics.

M31-6 well is an injection well, in December 1993 drilled and put into production, injection wells segment Es3 of 3307. 4-3422. 4m, total of 60.3m / 4 layer, injection method is a positive note, the pump pressure at $30 \mathrm{Mpa}$, oil pressure $30 \mathrm{Mpa}$, daily injection capacity of $10.81 \mathrm{~m}^{3}$, as of October 2015 the accumulated water $3.4926 \times 10^{4} \mathrm{~m}^{3}$, note the amount of water per day in October decreased to less than $2 \mathrm{~m}^{3}$, reach with injection requirements, October 20 stop Note acidizing, waiting for the next acidification.

\section{Technical Difficulties}

(1) The low permeability reservoir, in the development of performance as well productivity is low, drainage radius is small, limited conventional acidification radius;

(2) in the longitudinal direction of the reservoir performance for multi-development, strong heterogeneity, physical property was poor, the interlayer contradictions, easy to form secondary sedimentation conventional acidification, caused by the formation of new damage;

(3) the reservoir sensitivity is strong, injection water and formation incompatible produce expanded clay material, migration, formation pore blockage, caused by water pressure increases, reducing water capacity.

(4) Early Large soil acid and fluoboric acid fracturing technology on the one hand as a communication microfractures, corrosion holes and other migration channels; on the other hand excessive erosion near-wellbore to form a hole enlargement.

\section{Process scheme}

Consider the reservoir rock containing clay, quartz, feldspar and other substances, because driving fluid invaded the drilling and completion, workover and other operations, the reaction occurs with the formation of the original mineral, so that the original reservoir water swelling clay minerals and severe precipitation will generate a variety of chemicals clogged pores, allowing water difficulties, result is not water injection wells or injection is too small, this phenomenon of already having a water-sensitive nature of low permeability reservoir will be more serious ${ }^{[3]}$.

Acidification of mud acid is a sandstone reservoir by injection wells buck common technique is widely used in sandstone reservoir wells acidification, to restore or improve water reservoir, stable oil production played an important role ${ }^{[4]}$. However, due to soil reaction with acid clay minerals too fast, easy to damage the basic structure of the reservoir rock, and in severe cases can lead to the reservoir collapse, causing water problems worse.

Meanwhile, the reaction amount of soil less acid on silica matrix, can only be used to restore the original reservoir permeability and chemical reaction process may be related to $\mathrm{F}, \mathrm{SiF}$ fluoride salt precipitation and the formation of silicon fluoride salt precipitation and other secondary precipitation, resulting in new pollution.

And mud acid compared to more hydrogen acid is a new sandstone reservoir acidification acid system, using a special fluorine compound to replace $\mathrm{HCl}$ salt hydrogenation reaction occurs, can be decomposed multifunction acid under different stoichiometric conditions, with retarder resistance, strong adsorption, delay / suppression near wellbore precipitate generation ability. Can accelerate catalytic reaction rate of hydrofluoric acid and silica, and to achieve a uniform 
distribution of acid and acidified deep reservoir, without causing more damage to the formation ${ }^{[5]}$.

According to the reservoir characteristics of M31 block, pre-selection of hydrochloric acid added to achieve organic retarder, body acid may be using the new hydrogen-acidified retarder system, screened out for repeated acidification of the reservoir fluid system: HCL + XT-59 mixed acid + XT-92 multi-effect additives + XT-13 shrink swelling agent + XH-2 agents + XT-41 initiator + SP-33 inhibitor.

(1) sub-acid packer

Sub-acid packer role is to block the oil casing annulus, constraints acid according to the design channel choked to a ground to achieve single sealing card, acidizing single purpose. Sub-acid packer hydraulic packer and closed, in the construction without a separate packer, pressure tubing packer automatic packer. After acidification, relief tubing, packer released automatically.

(2) Acid spray device

Spray acid is role is to provide the acid from the tubing into the formation of the channel, sprayed acid is constant pressure by the sleeve and the opening device of dual control, only the sleeve is opened and the pressure exceeds the opening pressure, spray acid injection ports will open.

(3) Hydraulic anchor

Action hydraulic anchor is fixed string, string to prevent channeling during construction to ensure construction safety. Rely on hydraulic control hydraulic anchor, tubing crackdown, fluke to overcome the spring resistance radial stretch out, pressed on the inner wall of the casing, formation of anchorage force, fixed string. After the tubing pressure relief, automatic recovery fluke under spring pressure, the lifting anchor.

(4) Safety apparatus joint

Safety apparatus joint upper and lower joint composition, intermediate type buckle is buckle in the accident process can forward flip, raise the upper column.

(5) Wash wells valve

Wash well under the string when the valve sleeve communicating oil passage, when acidizing, the ball falling in this form a seal.

(6) Circulating valve

The role is to provide a wash cycle the valve shaft passage when starting acidification column for tubing relief.

General acidification due to formation heterogeneity effects, low permeability reservoir can not be effectively improved, resulting in more prominent contradiction between layers. We designed a new multi-stage stratified acidification string, it can achieve the transformation to a multi-layer system one by one, greatly improving the degree of transformation of acidification, targeted, well augmented injection effect.

The acidification well section: 3404.0-3422.4m, 18.4m / 1 layer; 3361.0-3374.2m, 13.2m / 1 layer; 3342.0-3348.5m, 6.5m / 1 layer; 3307.4-3329.6m, 22.2m / 1 layer.

Construction column (from the bottom) were circulatory located fittings + spray acid ( I grade) + K344-114 no packer sleeve ( I grade) + sleeve spray acid unit ( II grade) + D73.02mm outside upset tubing $3+$ K344-114 packer sleeve ( II grade) + sleeve spray acid unit (III level) + D73.02mm external upset tubing $2+$ K344-114 packer sleeve (III grade ) + sleeve spray acid unit (IV grade) + D73.02mm external upset tubing $3+$ K344-114 packer sleeve (IV grade) + hydraulic anchor + D73mm EUE tubing pup joints + safety apparatus joint + loop valve + D73.02mm external upset tubing $2+$ correl nipple + D73.02mm deep short section EUE tubing to the wellhead (Figure 1).

Working principle: by opening the sliding sleeve switch control packer setting and spray acid open step by step. When the first layer acidification levels I spray acid and I packer open, realize the top packer card underlying acidizing treatment; The second layer acidification, levels II spray acid and II packer open, I , II packer setting, achieve dual packer card monolayer acidizing treatment; The third layer with the second layer acidification, acidification when levels III packer after open and II packer to realize double seal card single-layer acidizing treatment. 
Fourth layer acidification acidification with the third layer, when levels IV packer after open and III packer to realize double seal card single-layer acidizing treatment.

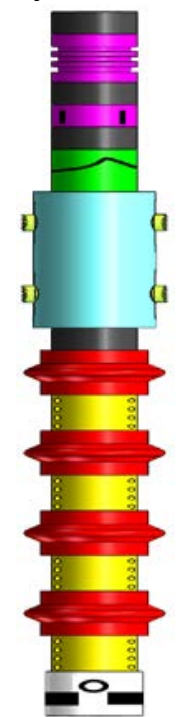

Fig.1. M31-6 string schematic construction

Features: multi-stage stratified trip string acidified liquid tubing can be rotated out, to prevent the liquid is extruded tubing formation, resulting reservoir contamination. Controllable, according to the pump pressure, casing pressure and displacement judgment packer packer, shear pins, sleeve open, spraying acid starts peers. After acidification, the open loop sleeve, the liquid was taken to avoid tubing wellhead, the wellhead to prevent contamination from drilling.

(1) The acidified stratified press tool design connector down into the well design position, adjust the packer packer design card points to the location.

(2) Construction of the first layer: the acidification wellhead injection cycle each gate adjustment positive status, pitching, acid injection pump cycle, returning the liquid into the tank. After the ball in place, the pump pressure rises, grade I packer packer, closed casing valve, the pressure continues to rise, grade I spray acid opens. I grade acid by spraying acid, oil casing annulus into the acidification layer 1.

The second layer of construction: put the second goal, pumping the ball to II grade packer sleeve, then the pump pressure and continue to hold back the pressure to cut short sleeve pin, ball and sliding sleeve down to II grade spray acid device, the acid is sprayed inside sleeve opens downward to form I level within blocked packer center tube. At this stage II open spray acid, the acid by spraying acid II grade, oil casing annulus passage into acidified layer 2.

The third layer construction: put the third ball, pumped to III grade packer, the packer level III and spray acid is successively sleeve and the inner sleeve ball down to II grade central tube formation packer open clogged. At this stage III packer packer, grade III open mouth spray acid, the acid by spraying acid III grade, oil casing annulus passage into acidified layer 3.

The fourth layer construction: the fourth put the ball, pumped to stage IV packer, packer will IV grade and spray acid is successively open ball sleeve and the inner sleeve down to center stage III packer tube formation blockage. At this stage IV packer packer, grade IV spray acid mouth open, IV grade acid by spraying acid, oil casing annulus passage into acidified layer 4.

Into the fifth ball, opening the circulating valve sleeve, when oil rises, the pressure to hold the valve sleeve pin cut cycle, cycle the valve is opened, tubing and casing annulus communication end acidizing.

(3) After construction is completed, the packer and re-opened. The reaction was acidified to be completed, immediately anti-handling, but also reverse circulation washing wells. 


\section{Field Application}

According to the construction design, on November 5, 2015 for a comprehensive water injection wells M31-6 curve more hydracid multilevel hierarchical acidification construction, the construction shown in Figure 2.

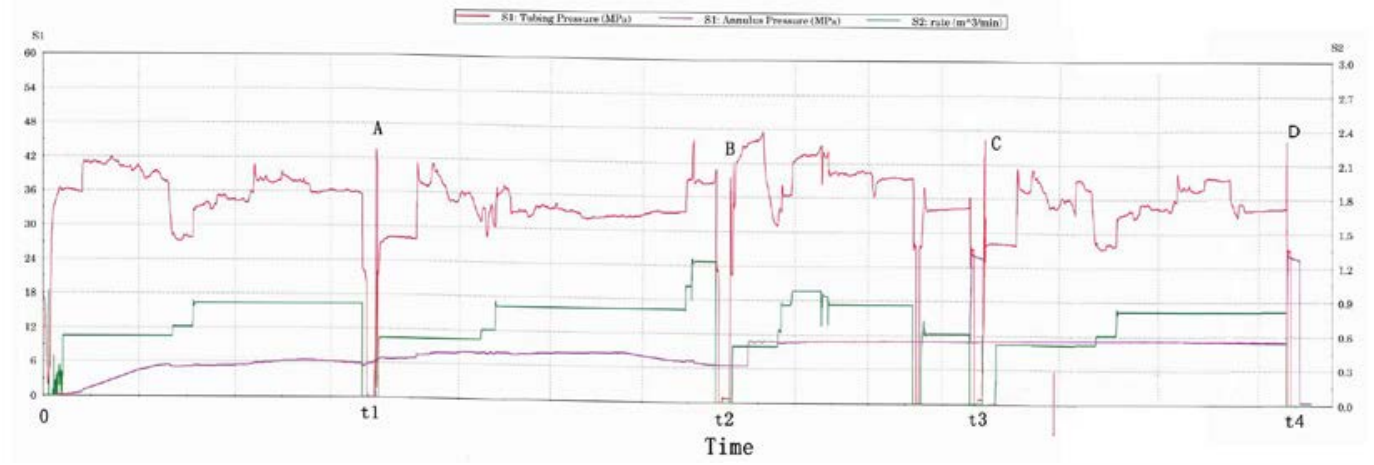

Fig.2. M31-6 construction well integrated hydrogen-acidified graph

Shown in Figure 2 red line is the oil pressure (MPa), the purple line is the casing pressure (MPa), the green line is the injection rate $\left(\mathrm{m}^{3} / \mathrm{min}\right)$. 0 - $\mathrm{t} 1$ for the first layer was acidified, $\mathrm{t} 1-\mathrm{t} 2$ for the second layer was acidified, t2-t3 for the third layer was acidified, t3-t4 of the fourth layer was acidified $\mathrm{t} 4$ after the recirculation valve is open pitch. Wherein A is II grade spray acid is instantaneous pressure sleeve open display point, B is grade III spray acid is instantaneous pressure sleeve open display points, $\mathrm{C}$ is the level IV spray acid is instantaneous pressure sleeve open display point, D point is circulating valve opens instantly, when casing pressure rising rapidly, oil sets connected.

Construction Difficulties: M31-6 well as injection wells, wellhead pressure, poor water absorption capacity, at $0.8 \mathrm{~m}^{3} / \mathrm{min}$ average emissions, construction pressures up to $48 \mathrm{MPa}$. Construction process casing pressure is stable, reliable packer packer. Acidizing smoothly, the various stages of the response curve distinctive characteristics. The results show that the multi-stage stratified high pressure injection wells acidification string acidizing meet requirements.

For October 2015 to November, water injection wells M31-6 statistics, shown in Figure 3.

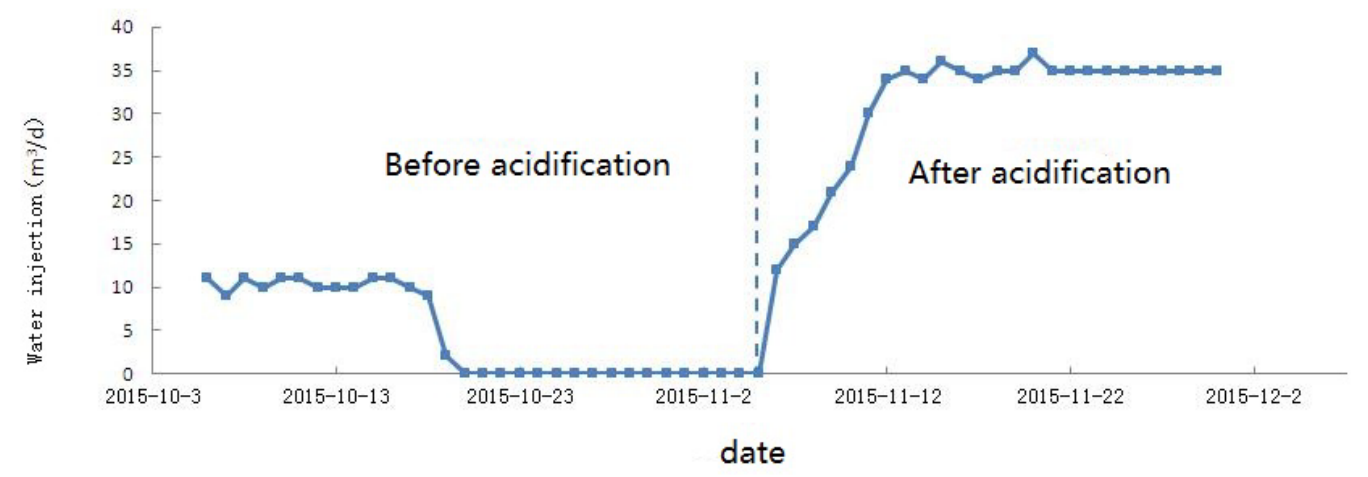

Fig.3. M31-6 day before and after acidizing water injection wells comparison chart

After M31-6 well as injection wells, November 5, 2015 implementation of the multi-layered hydrogen acid acidification, water pressure is reduced by the measures before 30MPa 22.4Mpa, to achieve the desired antihypertensive effect; the average daily water injection rate from less than before $10 \mathrm{~m}^{3}$, up to now the average daily water injection rate stabilized at around $35 \mathrm{~m}^{3}$, increasing injection effect is remarkable.

\section{Conclusion}

(1) By comparison with soil acid, the retarder performance of Multi-hydrogen acid is more suitable for sandstone reservoirs with high temperature, low permeability and high clay content. Multi-hydrogen acid can significantly reduce the acidification of the reaction rate, reduce the 
generation of secondary sedimentation, increase acidification distance, and improve acidification effect.

(2) Multi hydracid multilevel hierarchical acidification is time-saving, and the tool performance is reliable. This new process has larger acidification radius, and is highly targeted; the flowback rate is high after measures, and can effectively prevent the formation of secondary pollution.

(3) For M31-6 well with large water injection difference between layers, the technology can effectively avoid the interaction between different layers, and can reduce water injection pressure and increase water capacity significantly. The technology provides valuable experience for the repeat acidizing of the low permeability reservoir layers in the future.

\section{References}

[1] Zhang Ningxing. Multi-hydrogen Acid resolve blocking technology in Huanxiling Oilfield [J]. Oil Drilling \& Production Technology, 2010, 32 (6): 136-138.

[2] Yang Zi, Feng Weihua, Shi Yang. Application and Research on hydrogen-acid technology in offshore oil fields [J]. Petrochemical Industry Application, 2014, 33(2): 59-62.

[3] Jin Hongxia, Nie Qing, Zhang Yuting. Low permeability sandstone reservoir hydrogen-acid deep penetration acidification technology research and field testing [J]. Liaoning Chemical, 2011, 40(9): 989-992.

[4] Yang Jing, Wen Xiao, Ji Chaofeng. Application of hydrogen-acid technology Dagang Oilfield segment Duanliubo block blood pressure by injection [J]. Drilling \& Production Technology, 2008, 31(8): 63-67.

[5] Wang Wei, Xu Rongwu. Multi-hydrogen Acid Technology Application in Qinghai Oilfield Gasi [J]. Inner Mongolia Petrochemical Industry, 2010, 19(1): 124-126. 